\title{
Diagnostic Value of TB-IGRA, PPD, TB-DNA-PCR and TB-Ab in Silicosis Complicated with Tuberculosis
}

\author{
Weijia Lin1, Zhi Liu'², Yaping Zhang1, Feng Li1, Xiulong Zhang1, Zhihua Zhang1 \\ ${ }^{1}$ Department of Respiratory Medicine, The First Affiliated Hospital of Hebei North University, Zhangjiakou, China \\ ${ }^{2}$ Department of Orthopaedics, The First Affiliated Hospital of Hebei North University, Zhangjiakou, China \\ Email: lwj1987lu@163.com, *liuzhi309@126.com
}

How to cite this paper: Lin, W.J., Liu, Z., Zhang, Y.P., Li, F., Zhang, X.L. and Zhang, Z.H. (2021) Diagnostic Value of TB-IGRA, PPD, TB-DNA-PCR and TB-Ab in Silicosis Complicated with Tuberculosis. Journal of Tuberculosis Research, 9, 110-116. https://doi.org/10.4236/jtr.2021.92010

Received: May 27, 2021

Accepted: June 25, 2021

Published: June 28, 2021

Copyright ( 2021 by author(s) and Scientific Research Publishing Inc. This work is licensed under the Creative Commons Attribution International License (CC BY 4.0).

http://creativecommons.org/licenses/by/4.0/

\begin{abstract}
Purpose: The purpose of this article is to investigate the clinical value of TBIGRA (Tuberculosis-Interferon Gamma Release Assay), PPD (Intradermal Terbuculin Test), TB-DNA-PCR (Tuberculosis-Deoxyribonucleic-Polymerase Chain Reaction) and TB-Ab (Tuberculosis-Antibody) in diagnosing silicosis complicated with pulmonary tuberculosis. Methods: 53 cases of suspected silicosis complicated with pulmonary tuberculosis were selected in the time span ranging from February 2017 to May 2019. TB-IGRA test, PPD test, TBDNA-PCR and TB-Ab detection were performed. The sensitivity, specificity, positive predictive value and negative predictive value were calculated. $\mathrm{Re}$ sults: Silicosis and pulmonary tuberculosis were diagnosed in 11 cases, with an incidence of $20.75 \%$. The positive rates of TB-IGRA, PPD, TB-DNA-PCR and $\mathrm{TB}-\mathrm{Ab}$ were $66.04 \%, 30.19 \%, 5.67 \%$ and $26.42 \%$, respectively. The sensitivity was $90.91 \%, 81.82 \%, 27.27 \%$ and $54.55 \%$ respectively. The specificity was $42.86 \%, 80.95 \%, 100 \%$ and $80.95 \%$ respectively. The positive predictive values were $28.57 \%, 50 \%, 100 \%$ and $42.86 \%$ respectively. The negative predictive values were $94.44 \%, 91.89 \%, 84 \%$ and $87.18 \%$. The positive rate, sensitivity and negative predictive value of TB-IGRA were the highest, while the specificity of TB-DNA-PCR was the highest yet with low positive rate, sensitivity and positive predictive value. Conclusion: The positive rate and sensitivity of TB-IGRA were high, yet with poor specificity, so it was impossible to judge whether the cases belonged to active pulmonary tuberculosis. The combination of PPD and TB-DNA-PCR could improve the sensitivity, specificity and positive predictive value, and the diagnostic accuracy of active pulmonary tuberculosis, which showed satisfactory clinical value.
\end{abstract}

\section{Keywords}

TB-IGRA, PPD, TB-DNA-PCR, TB-Ab, Diagnosis 


\section{Introduction}

In recent years, with the economic globalization, the widening gap between the rich and the poor and the increase of rural-urban population flow, the incidence of tuberculosis represents an increasing trend, which has seriously threatened people's physical and mental health. The medical services of our hospital has extended to Hebei, Shanxi, Inner Mongolia three provinces; as we all know that the mining industry and metal smelting industry in the above three provinces are relatively developed, there is a large proportion of mining workers in these three areas, which has resulted in a high incidence of silicosis. Studies have confirmed that silicosis patients are prone to secondary pulmonary tuberculosis, leading to an increase in the incidence of pulmonary tuberculosis [1], Therefore, the situation of tuberculosis prevention and control is still challenging, which has been still considered as the focus of public health issues. The tuberculosis focus is easily wrapped or even overlapped by silicosis nodules, which leads to low positive rate of TB in traditional methods. TB-IGRA technology is the up-to-date in vitro immunodiagnostic method for TB infection worldwide. It has been widely used in North America and Europe. In 2011, it was approved in China [2] [3]. The basic principle is: The T lymphocytes produced by the body infected with Mycobacterium tuberculosis will release $\gamma$ interferon when stimulated by specific antigen in vitro. Therefore, the quantitative analysis of the $\gamma$ interferon is capable of determining whether there is a specific $\mathrm{T}$ cell response related to Mycobacterium tuberculosis. Large data samples suggested that TB-IGRA had high sensitivity, accuracy and specificity in TB infection diagnosis. However, since the traditional PPD, TB-DNA-PCR, TB-Ab detection methods are low in sensitivity, it is urgent to find faster methods with higher sensitivity, specific for diagnostic of silicosis complicated with tuberculosis [4] [5].

\section{Materials and Methods}

\subsection{Materials}

From February 2017 to May 2019, 53 cases of suspected silicosis complicated with pulmonary tuberculosis were selected.

\subsection{Inclusion and Exclusion Criteria}

Inclusion criteria: 1) The cases were in accordance with GBZ70-2009 "diagnostic criteria for pneumoconiosis" [6]; 2) Recently, the patient had fever, night sweats, cough, hemoptysis, wheezing, pleural effusion and other suspected tuberculosis symptoms; 3) They did not receive Isoniazid or Rifampicin or Rifapentine or Ethambutol or other antituberculosis treatment; 4) The patients agreed to be enrolled in the study and performed active coorperation.

Exclusion criteria: 1) Patients were diagnosed with other diseases, such as lung cancer, pneumonia, pulmonary abscess, etc.; 2) Patients who have combined with immune system diseases, heart failure, respiratory failure and critical illness; 3) Patients who have received antituberculosis treatment; 4) Patients and their 
families refused to participate in the study, cannot actively cooperate with the inspection.

\section{Methods}

All patients received four kinds of tuberculosis detection: TB-IGRA,TB-Ab, PPD, TB-DNA-PCR.

\subsection{TB-IGRA}

In the early morning, $6-10 \mathrm{~mL}$ of fasting peripheral venous blood was extracted and was anticoagulated with heparin, and peripheral monocytes were isolated. 50 $\mu \mathrm{L}$ of cell culture medium, ESAT- 6 (antigen A), CFP-10 (antigen B) and positive control solution were added to 4 wells in microplate respectively (blank control, test hole A, test hole B and positive control), followed by adding $100 \mu \mathrm{L}$ samples per well. The microplate was then incubated in incubator. The microplate was washed with PBS and enzyme labeled antibody was added. The microplate was washed again and substrate reaction solution was added. ELISPOT reader was utilized to read and count for the number of spots. The result was rigorously assessed under the instruction of the kit. If the number of negative spots is $0-5$, and the number of antigen $\mathrm{A}$ or antigen $\mathrm{B}$ spots subtracted by the number of negative spots is above 6 , the result will be judged as positive. If the number of negative spots is above 6 , and the number of detective spots is more than 2 times of the number of negative spots, the result will also be judged as positive; if the result doesn't measure up the above standards and the positive control hole is normal, the result will be regarded as negative.

\subsection{TB-Ab}

Colloidal gold immunochromatography was performed to determine serum tuberculosis antibody. The reagent was provided by Shanghai Aopu biological company, and the operation was carried out according to the instruction of the kit.

\subsection{PPD}

After subcutaneous injection of $5 \mathrm{IU}$ tuberculosis protein derivative into the inner site of left forearm for 72 hours, the average diameter of induration was observed and calculated by (transverse diameter + longitudinal diameter)/2. When there is no induration or the average diameter of induration is less than $10 \mathrm{~mm}$, the result will be regarded as negative (-); and the result will be regarded as positive $=(+)$ when the diameter exceeds $10 \mathrm{~mm}$.

\subsection{Sputum or Pleural Effusion TB-DNA-PCR}

After gargling with clear water in the early morning, deep expectoration or drainage of pleural effusion were gathered in sterile sputum box for examination. TB-DNA detection kit of Sun Yat-sen University Da'an gene Co., Ltd. was used 
and operated according to the instruction.

\section{Observational Index}

Sensitivity, specificity, positive predictive value and negative predictive value of TB-IGRA, PPD, TB-DNA-PCR, TB-Ab were observed.

Sensitivity: percentage of positive samples in the total number of diagnosed patients.

Specificity: percentage of negative samples in the total number of ruled-out patients.

Positive predictive value: percentage of diagnosed patients in the total number of positive samples.

Negative predictive value: percentage of ruled-out patients in the total number of negative samples.

\section{Statistical Method [7] [8]}

Spss20.0 statistical software was used to process the data. Measurement data was presented by $\bar{x} \pm \mathrm{S}$; Count data was expressed in \%.

\section{Results}

\subsection{General Results}

There were 52 males and 1 female in 53 cases of suspected silicosis complicated with pulmonary tuberculosis, aged from 42 to 86 years old, with an average age of $(61.851 \pm 11.32)$. 11 cases were diagnosed as pulmonary tuberculosis, and the incidence rate was $20.75 \%, 42$ cases were ruled out of tuberculosis, and the 11 cases were given anti tuberculosis drugs intervention (Table 1).

\subsection{Positive and Negative Rates of TB-IGRA, PPD, TB-DNA-PCR and TB-Ab}

A total of 53 cases were included in this study, among which 11 cases were diagnosed as pulmonary tuberculosis, and 42 cases were ruled out of tuberculosis. The number of TB-IGRA positive cases was 35 (66.04\%), and negative cases was 18 (33.96\%). 16 cases (30.19\%) were PPD positive and 37 cases $(69.81 \%)$ were PPD negative. TB-DNA-PCR was positive in 3 cases (5.67\%) and negative in 50 cases (94.33\%). 14 cases (26.42\%) were TB-Ab positive and 39 cases $(73.58 \%)$ were $\mathrm{TB}-\mathrm{Ab}$ negative (Table 2).

\subsection{Sensitivity, Specificity, Positive Predictive Value and Negative Predictive Value of TB-IGRA, PPD, TB-DNA-PCR and TB-Ab}

Sensitivity, specificity, positive predictive value and negative predictive value of TB-IGRA were $90.91 \%, 42.86 \%, 28.57 \%$ and $94.44 \%$, respectively. Sensitivity, specificity, positive predictive value and negative predictive value of PPD were $81.82 \%, 80.95 \%, 50 \%$ and $91.89 \%$, respectively. Sensitivity, specificity, positive predictive value and negative predictive value of TB-DNA-PCR were $27.27 \%$, 
$100 \%, 100 \%$ and $84 \%$, respectively. Sensitivity, specificity, positive predictive value and negative predictive value of TB-Ab were $54.55 \%, 80.95 \%, 42.86 \%$ and $87.18 \%$, respectively. Among them, TB-IGRA possessed the highest sensitivity and negative predictive value, while TB-DNA-PCR had the highest specificity and positive predictive value (Table 3 ).

\section{Discussion}

Tuberculosis is the most common complication of silicosis. Meta analysis [9] conducted by mirsadraee et al. showed that mobidity of tuberculosis in pneumoconiosis patients was $22.50 \%$, which was significantly higher than that of normal people (6.3\%). At present Silicosis is a common occupational disease in China, and patients are at high risk of developing tuberculosis. According to statistics, the rate of developing tuberculosis in stage I silicosis is about $18.9 \%$, stage II is $33.3 \%$, and stage III is $84.8 \%$. What's more, silicosis and tuberculosis promote each other and accelerates the deterioration of the disease. Tuberculosis is a chronic infectious disease caused by Mycobacterium tuberculosis, some of which are latent infection or hidden infection, and the clinical manifestations are not

Table 1. General information of participants.

\begin{tabular}{|c|c|c|c|c|c|c|c|c|}
\hline & Case (s) & Diagnose & Age & Average age & Clinical symptoms & Coalmine & Gold ore & Quartz \\
\hline \multirow[t]{6}{*}{ Male } & 52 & 11 & $42-86$ & $61.85 \pm 11.32$ & yes $52 \quad$ no 0 & 23 & 20 & 9 \\
\hline & & & & & fever 8 & & & \\
\hline & & & & & night sweats 4 & & & \\
\hline & & & & & cough 42 & & & \\
\hline & & & & & wheezing 50 & & & \\
\hline & & & & & pleural effusion 13 & & & \\
\hline Female & 1 & 0 & 66 & 66 & yes 0 & & & 1 \\
\hline
\end{tabular}

Table 2. Positive rate and negative rate of four tuberculosis detection indexes.

\begin{tabular}{cccc}
\hline & TB-IGRA & PPD & TB-DNA-PCR \\
\hline Number of positive cases & 35 & 16 & 3 \\
Number of negative cases & 18 & 37 & 50 \\
Positive rate & $66.04 \%$ & $30.19 \%$ & $5.67 \%$ \\
Negative rate & $33.96 \%$ & $69.81 \%$ & $94.33 \%$ \\
\hline
\end{tabular}

Table 3. Sensitivity, specificity, positive predictive value and negative predictive value of the four tuberculosis detection indexes $\%$.

\begin{tabular}{|c|c|c|c|c|}
\hline & TB-IGRA & PPD & TB-DNA-PCR & $\mathrm{TB}-\mathrm{Ab}$ \\
\hline Sensitivity & $90.91(10 / 11)$ & $81.82(8 / 11)$ & $27.27(3 / 11)$ & $54.55(6 / 11)$ \\
\hline Specificity & $42.86(18 / 42)$ & $80.95(34 / 42)$ & $100(42 / 42)$ & $80.95(34 / 42)$ \\
\hline Positive predictive value & $28.57(10 / 35)$ & $50(8 / 16)$ & $100(3 / 3)$ & $42.86(6 / 14)$ \\
\hline Negative predictive value & $94.44(17 / 18)$ & $91.89(34 / 37)$ & $84(42 / 50)$ & $87.18(34 / 39)$ \\
\hline
\end{tabular}


specific. Traditional diagnostic methods of tuberculosis are poor in sensitivity and specificity, for example, culturing Mycobacterium tuberculosis takes a long time and the low positive rate is low, while false positive or false negative is easy to see in tuberculin test. TB-IGRA is a novel method to diagnose tuberculosis developed in recent years. It has been demonstrated to have good sensitivity and specificity in detecting Mycobacterium tuberculosis infection. It is expressed early in vivo, before the appearance of bacteriological evidence and typical imaging manifestations which permits early diagnosis and has become popular in Europe and America. The mechanism of TB-IGRA detection [10] is that upon infection of Mycobacterium tuberculosis, T-lymphocytes were stimulated to produce. When physically stimulated by the tuberculin again, interferon will be secreted immediately. ELISPOT is used to count interferon- $\gamma$ peripheral blood mononuclear cells, and then diagnostic conclusion was drawn.

11 cases of pulmonary tuberculosis were diagnosed in this study, with the incidence of $20.75 \%$. Positive rates of TB-IGRA, PPD, TB-DNA-PCR and TB-Ab were $66.04 \%, 30.19 \%, 5.67 \%$ and $26.42 \%$, with the sensitivity of $90.91 \%, 81.82 \%$, $27.27 \%$ and $54.55 \%$, the specificity of $42.86 \%, 80.95 \%, 100 \%$ and $80.95 \%$. The positive predictive values of $28.57 \%, 50 \%, 100 \%$ and $42.86 \%$ and the negative predictive values of $94.44 \%, 91.89 \%, 84 \%$ and $87.18 \%$, respectively. TB-IGRA was highest in positive rate, sensitivity and negative predictive value, while TB-DNA-PCR had the highest specificity, with low positive rate, sensitivity and positive predictive value. The values of PPD and TB-Ab were between the two. PPD was high in sensitivity and specificity, but clinical strong positive value is low and is relatively high in false-positive rate and false-negative rate.

To sum up, TB-IGRA is high in positive rate and sensitivity, which is better than the traditional sputum examination of tuberculosis. Yet with poor specificity, it's unable to judge whether the case is active tuberculosis, which is limited in clinical diagnosis. However, it can be used as an auxiliary diagnostic item. Combination of PPD and TB-DNA-PCR can improve the sensitivity, specificity and positive predictive value, and also improve the diagnostic accuracy of active tuberculosis, which can make up for each other's shortcomings, thus has favorable clinical value in the auxiliary diagnosis of active tuberculosis.

\section{Project Name}

The project name is 2018 key project plan of medical science research in Hebei Province 20180869 Comparison between TB-IGRA detection and bronchoalveolar lavage fluid tuberculosis detection in the diagnosis of silicosis with pulmonary tuberculosis.

\section{Conflicts of Interest}

The authors declare no conflicts of interest regarding the publication of this paper.

\section{References}

[1] Wang, R.-F. (2021) Clinical Characteristics of Senile Pneumoconiosis. Chinese Jour- 
nal of Industrial Medicine, 34, 176-178.

[2] Pang, C., Wu, Y., Wan, C., et al. (2016) Accuracy of the Bronchoalveolar Lavage Enzyme-Linked Immunospot Assay for the Diagnosis of Pulmonary Tuberculosis: A Meta-Analysis. Medicine, 95, e3183. https://doi.org/10.1097/MD.0000000000003183

[3] Cattamanchi, A., Ssewenyana, I., Nabatanzi, R., et al. (2012) Bronchoalveolar Lavage Enzyme-Linked Immunospot for Diagnosis of Smear-Negative Tuberculosis in HIV-Infected Patients. PLoS ONE, 7, e39838. https://doi.org/10.1371/journal.pone.0039838

[4] Zhang, L.-H. (2021) $\gamma$-Diagnostic Efficacy of Interference Sample Release Test in Tuberculosis Patients of Different Ages. Medical Equipment, 34, 50-51. (In Chinese)

https://kns.cnki.net/kcms/detail/detail.aspx?dbcode=CJFD\&dbname=CJFDLAST20 21\&filename $=$ YLZB202105022\&v=t\%25mmd2FuueDfgei\%25mmd2FFYziOn75nDtl T94HXvU4EeA8\%25mmd2F3zyMEnl2bv8k2Ht3Jlj2zVN9RpX

[5] Huang, Y.-N. (2021) Diagnostic Value of Parallel Detection of T-SPOT.TB, TB Sputum Smear and TB-DNA in Tuberculosis. Heilongjiang Medicine Journal, 34, 455-457. (In Chinese)

https://kns.cnki.net/kcms/detail/detail.aspx?dbcode=CJFD\&dbname=CJFDLAST20 21\&filename=HJYY202102091\&v=OSWWMdgr6sNIP9fclKdltTJySMDbS5NS4rLX U6Jz34tfMlljARdF9Go\%25mmd2BFdZNJ4vl

[6] GBZ70 (2009) Diagnostic Criteria of Pneumoconiosis. (In Chinese) https://max.book118.com/html/2019/0108/5220133134002000.shtm

[7] Li, L.-J. (2021) Clinical Significance of Combined Detection of T-SPOT.TB, PPD and TB-AB in the Diagnosis of Active Pulmonary Tuberculosis. Occupational Health and Damage, 36, 106-109.

[8] Zheng, Y., Xu, D.-M. and Ding, W.-J. (2016) Comparison of Diagnostic Value of Four Different Detection Methods for Pulmonary Tuberculosis. Chinese Journal of Rural Medicine and Pharmacy, 23, 65-66.

[9] Mirsadraee, M., Saffari, A., Sarafraz, M., et al. (2013) Frequency of Tuberculosis in Anthracosis of the Lung: A Systematic Review. Archives of Iranian Medicine, 16, 661-664.

[10] Jian, S.-N. and Qu, J.-M. (2012) The Value of T-SPOT.TB in the Diagnosis of Tuberculosis. International Journal of Respiration, 32, 439-442. (In Chinese) https://kns.cnki.net/kcms/detail/detail.aspx?dbcode=CJFD\&dbname=CJFDZHYX\& filename $=$ GWHX201206018\&v=vN9DMe3ez4VmeltyO4nc6nf0g5jns2jiJbDoBcNl6dBh kaTR8S\%25mmd2BlMOWADAaOEFLq 